\title{
Cell surface carbohydrates of symbiotic dinoflagellates and their role in the establishment of cnidarian-dinoflagellate symbiosis
}

\author{
Giada Tortorelli (iD) ${ }^{1 凶}$, Carsten Rautengarten ${ }^{1}$, Antony Bacic ${ }^{2}$, Gabriela Segal ${ }^{3}$, Berit Ebert ${ }^{1}$, Simon K. Davy (iD ${ }^{4}$, \\ Madeleine J. H. van Oppen (iD) ${ }^{1,5}$ and Geoffrey I. McFadden ${ }^{1}$
}

(C) The Author(s) 2021

\begin{abstract}
Symbiodiniaceae algae are often photosymbionts of reef-building corals. The establishment of their symbiosis resembles a microbial infection where eukaryotic pattern recognition receptors (e.g. lectins) are thought to recognize a specific range of taxonspecific microbial-associated molecular patterns (e.g. glycans). The present study used the sea anemone, Exaiptasia diaphana and three species of Symbiodiniaceae (the homologous Breviolum minutum, the heterologous-compatible Cladocopium goreaui and the heterologous-incompatible Fugacium kawagutii) to compare the surface glycomes of three symbionts and explore the role of glycan-lectin interactions in host-symbiont recognition and establishment of symbiosis. We identified the nucleotide sugars of the algal cells, then examined glycans on the cell wall of the three symbiont species with monosaccharide analysis, lectin array technology and fluorescence microscopy of the algal cell decorated with fluorescently tagged lectins. Armed with this inventory of possible glycan moieties, we then assayed the ability of the three Symbiodiniaceae to colonize aposymbiotic E. diaphana after modifying the surface of one of the two partners. The Symbiodiniaceae cell-surface glycome varies among algal species. Trypsin treatment of the alga changed the rate of B. minutum and C. goreaui uptake, suggesting that a protein-based moiety is an essential part of compatible symbiont recognition. Our data strongly support the importance of D-galactose (in particular $\beta$-D-galactose) residues in the establishment of the cnidarian-dinoflagellate symbiosis, and we propose a potential involvement of L-fucose, D-xylose and D-galacturonic acid in the early steps of this mutualism.
\end{abstract}

The ISME Journal (2022) 16:190-199; https://doi.org/10.1038/s41396-021-01059-w

\section{INTRODUCTION}

Symbiodiniaceae are single-celled alveolate protists of the phylum Dinoflagellata. Dinoflagellates inhabit temperate and tropical areas as phototrophs, heterotrophs, parasites and symbionts, but the Symbiodiniaceae are most widely recognized as intracellular photosymbionts of reef-building corals [1]. Coral-Symbiodiniaceae symbiosis is a mutualism where the symbiont benefits from inorganic nutrients coming from host metabolism, and the coral host receives a large proportion of photosynthate produced by the dinoflagellates, thus providing the coral partner with most of its energy requirements and therefore underpinning the entire coral reef ecosystem [2].

The family Symbiodiniaceae comprises at least nine genera $[1,3]$. The physiological differences among the genera-and even within genera-are enormous [4], and dramatically influence the physiology of the coral holobiont (i.e. the coral and its associated microbiota) and its ability to cope with environmental stressors [5]. Indeed, it is pivotal for the coral host to engage in symbiosis with specific symbiont types to survive [6].

The majority of coral species have a horizontal transmission mode and must acquire suitable symbionts from the surrounding environment $[7,8]$. Symbiosis establishment via horizontal mechanisms involves attraction, recognition and uptake of 'the right' symbionts [2]. Although there is some knowledge about the processes of attraction [9, 10] and uptake [11, 12, 13], little is known on the mechanisms of symbiotic specificity and host-symbiont selection.

Inter-partner signalling molecules involved in symbiosis establishment between corals and symbiotic dinoflagellates are still being explored [14]. The initiation of this symbiosis has been compared to the recognition of a pathogen during microbial infection [15]. In this model, the molecular crosstalk between the two symbiotic partners is mediated by the specific interaction between taxon-specific microbial-associated molecular patterns (MAMPs) and pattern recognition receptors (PRRs). A widespread MAMP-PRR system is the glycan-lectin interaction that acts as a lock-and-key mechanism in both beneficial and detrimental symbioses [16], and this is proposed as a model for establishment of the cnidarian-dinoflagellate mutualism [15].

The cell surface of symbiotic dinoflagellates is populated with glycoconjugates, with some glycan motifs similar among species and others unique to each species [17]. Two types of sugar linkages, mannose-mannose and galactose- $\beta(1-4)-\mathrm{N}$-acetylglucosamine, have been reported in the algal cell walls [18]. Conversely,

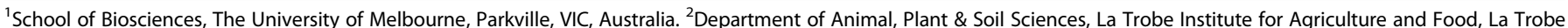

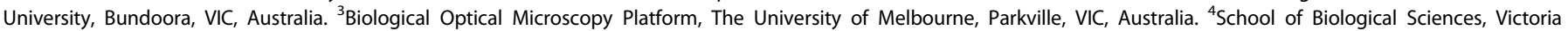
University of Wellington, Wellington, New Zealand. ${ }^{5}$ Australian Institute of Marine Science, Townsville, QLD, Australia. ${ }^{凶}$ email: giadatortorelli@gmail.com
} 
mannose-binding lectin (Millectin; [19]), two N-acetyl-Dgalactosamine binding proteins (Tachylectin-2-like lectin AtTL-2; [20]) and two D-galactose binding lectins, SLL-2 [21] and CecL [22], have been isolated from several coral species and shown to be involved in various phases of symbiosis establishment. The welldescribed N-glycan biosynthesis pathway in yeast, plants and humans [23] is also present in Symbiodiniaceae and is a key factor in cnidarian-dinoflagellate symbiosis establishment, highlighting the importance of the symbiont's cell-surface sugar composition in this process. The majority of the N-glycan types found on the symbiont surface were mannose-rich, further supporting the role of mannose in symbiosis onset [24]. The sugars N-acetyl-Dgalactosamine [20, 25], fucose [24] and a-glucose [25, 26] were also found on the symbiont cell surface. Several studies thus support the involvement of glycan-lectin interactions in the establishment of symbiosis between cnidarians and Symbiodiniaceae, but their roles require further exploration.

Here, we adopted a model organism approach to explore the molecules at the interface of the cnidarian-dinoflagellate symbiosis and investigate the hypothesis that a glycan-lectin interaction plays a role in the initiation of this relationship. The model is the sea anemone, Exaiptasia diaphana, which forms symbioses with members of the Symbiodiniaceae [15]. Importantly, E. diaphana can be freed of all symbionts by chemical bleaching, and then inoculated with new symbionts, thus providing an ideal platform to dissect host/symbiont recognition at a molecular level. We previously developed a matrix of host-symbiont compatibilities [27] with homologous-compatible (Breviolum minutum), heterologous-compatible (Cladocopium goreaui) and incompatible (Fugacium kawagutii) symbionts and three different anemone genotypes [28]. We hypothesized that the compatible symbionts (B. minutum and C. goreaui) have similar cell-surface structures that fit the lock-and-key mechanism of the host and are different from the ones on the cell surface of incompatible algae ( $F$. kawagutii). Based on this concept, we explored and compared the composition of the three Symbiodiniaceae cell surfaces. A multi-step approach, comprising both well-established [20, 29, 30] and innovative methods [31], was adopted to profile the cell surface of these Symbiodiniaceae. In this way, we created a list of potential molecular candidates, that we then strategically altered to investigate their role(s) during the first stages of symbiosis.

\section{MATERIALS AND METHODS Experimental organisms}

Three anemone genotypes (AIMS2, AIMS3 and AIMS4; [28]) of the cnidarian model E. diaphana sourced from the Great Barrier Reef (GBR) were used in this study. Polyps were kept in reconstituted seawater (RSS, [27]) in a growth chamber (LE-509, Thermoline Scientific) under constant temperature $\left(27^{\circ} \mathrm{C}\right), 12: 12$-h light:dark photoperiod cycle and 15- $\mu \mathrm{mol}$ photons $\mathrm{m}^{-2} \mathrm{~s}^{-1}$ irradiance (white + red LED lights, EDOLED), and fed with freshly hatched Artemia sp. nauplii ad libitum twice per week.

Three Symbiodiniaceae species were used in this work: $B$. minutum (MMSF 01, ITS2 type B1), C. goreaui (SCF 055-01.10, ITS2 type C1) and F. kawagutii (SCF 089.01, ITS2 type F1). These algae were chosen based on their different compatibilities with the anemone host [27]. The B. minutum culture was isolated from the $E$. diaphana anemones mentioned above [27], while C. goreaui and F. kawagutii were obtained from the Australian Institute of Marine Science (AIMS) where they were originally isolated from the GBR-sourced corals Acrocopora tenuis and Pocillopora damicornis, respectively. All cultures were maintained in 1-L Schott bottles with $0.2-\mu \mathrm{m}$ membrane vented caps in a growth chamber (740FHC LED, HiPoint) under constant temperature $\left(27^{\circ} \mathrm{C}\right), 12: 12$-h light:dark photoperiod cycle, and 60 $\mu \mathrm{mol}$ photons $\mathrm{m}^{-2} \mathrm{~s}^{-1}$ of light.

\section{Nucleotide sugar and monosaccharide component analysis of Symbiodiniaceae cell walls}

The nucleotide sugar and cell-wall monosaccharide compositions of $B$. minutum, $C$. goreaui and F. kawagutii were profiled using methods well established in higher plants $[31,32,33]$. For the nucleotide sugar analysis, Symbiodiniaceae cultures were harvested after $5 \mathrm{~h}$ of exposure to light. Fifty milligrams of algae per sample were resuspended in ice-cold methanol/chloroform (1:1 mixed solution), transferred into custom lysing matrix D 2-ml tubes (MP Biomedicals), ground in liquid nitrogen using a cryomill, then vortexed and placed at $-20^{\circ} \mathrm{C}$ for $2 \mathrm{~h}$. Following the addition of $400-\mu \mathrm{L}$ ice-cold water, the samples were centrifuged at $20,000 \times g$ at $4{ }^{\circ} \mathrm{C}$ and the upper phase was transferred into $15-\mathrm{ml}$ tubes placed on ice. The addition of ice-cold water and centrifugation steps were repeated two more times, and the supernatants were combined with the previously collected aqueous phase. Samples were frozen in liquid nitrogen and freeze-dried overnight. Sample purification was undertaken by solid phase extraction, then nucleotides sugars were detected and quantified by liquid chromatography tandem mass spectrometry (LC-MS/MS) using a 4,000 QTRAP LC/MS/MS system (SCIEX) equipped with a Turbolonspray source (SCIEX) and an Agilent 1,100 Series Capillary LC System. The following nucleotide sugar standards

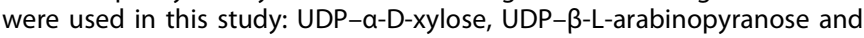
UDP-a-D-galacturonic acid (Carbosource Services, Complex Carbohydrate Research Center); UDP-a-D-glucuronic acid, UDP-a-D-glucose, UDP-a-D-galactose, UDP-N-acetyl-a-D-glucosamine, UDP-N-acetyl-a-Dgalactosamine, GDP- $\alpha$-D-mannose, GDP- $\beta$-L-fucose, GDP-Glc (SigmaAldrich) and UDP- $\beta$-L-arabinofuranose (Peptides International; [32]). Data were acquired using Analyst 1.5.1 (SCIEX) and nucleotide sugars were quantified using MultiQuant 2.1 software (SCIEX) by linear regression of the peak areas [33].

To analyse the algal cell-wall monosaccharide composition, we took 300 mg of pelleted algae, and ground the cells in liquid nitrogen as described above. To obtain cell-wall alcohol insoluble residues (AIR), the homogenate was incubated in $100 \% \mathrm{EtOH}$ at $100^{\circ} \mathrm{C}$ for 30 min under constant shaking at $1,400 \mathrm{rpm}$. The AIR was then pelleted by centrifugation at $20,000 \times g$ for $5 \mathrm{~min}$. The pellet was washed twice in $70 \% \mathrm{EtOH}$ and subsequently in acetone, and then left to dry overnight. Single sugar units (monosaccharides) were released by hydrolysis of AIRs with 2-N trifluoroacetic acid (TFA) and incubation at $120^{\circ} \mathrm{C}$ for $1 \mathrm{~h}$. The TFA solution was removed by evaporation overnight in a vacuum concentrator. The monosaccharide analysis was performed using High Performance Anion Exchange Chromatography coupled with Pulsed Amperometric Detection (HPAECPAD). Three replicates for each Symbiodiniaceae species were diluted and subsequently analysed on an ICS 6,000 (Dionex Corporation, Sunnyvale, CA) essentially as described in Rautengarten et al. 2014 [32], with the exception that the first isocratic elution step was performed with $4 \mathrm{mM}$ to separate xylose and mannose, and while in a separate run $8 \mathrm{mM}$ was used to separate $\mathrm{N}$-acetyl-galactosamine and rhamnose. Standards comprised $\mathrm{L}$ fucose, L-rhamnose, L-arabinose, C-galactose, D-galactose, D-xylose, Dgalacturonic acid, D-glucuronic acid, N-acetylgalactosamine and $\mathrm{N}$ acetylglucosamine. Both $\mathrm{N}$-acetylgalactosamine and $\mathrm{N}$-acetylglucosamine were TFA-treated prior to the run. A run of a standard mixture was performed with each sample set to enable sample quantitation by linear regression.

A one-way Anova (or a non-parametric Kruskal-Wallis test if normality criteria were not met) was performed to compare the relative amount of all monosaccharides found within a Symbiodiniaceae species and of each individual monosaccharide among B. minutum, C. goreaui and F. kawagutii. Pairwise comparisons were performed post hoc with Tukey's tests or nonparametric Dunn test $(p>0.05)$.

\section{Confocal microscopy of Symbiodiniaceae cell surface}

Fluorescent molecular probes conjugated with eight different lectins were used to explore their affinity for the glycoconjugates populating the cell surface of $B$. minutum, C. goreaui and F. kawagutii. Symbiodiniaceae cultures were divided into four aliquots of $1.5 \times 10^{6}$ cells for each species and treatment. The pelleted cells were resuspended in $4 \%$ paraformaldehyde (PFA)/ $1 \times$ PBS, fixed for $24 \mathrm{~h}$ at $4{ }^{\circ} \mathrm{C}$, washed in $1 \times \mathrm{PBS}$ and stored at $4{ }^{\circ} \mathrm{C}$. Three days later, each sample was washed $(4,000 \times g)$ three times in $1 \times$ PBS, then one of the fluorescent lectins was added to the algal solution at a final concentration of $1 \mathrm{mg} / \mathrm{ml}$ [29]. The fluorescent lectin conjugates used in this study, and their glycan affinities, are described in Table 1. After a 1-h incubation at room temperature in the dark, samples were washed three times in $1 \times$ PBS and cells resuspended in a final volume of $100 \mu$ l. Five microliters of each sample were placed in four wells of a teflon printed microscope slide (ProSciTech), and then mounted with ProLong gold antifade mountant (ThermoFisher scientific). Unstained controls were also prepared to profile the innate fluorescence 
of all algal strains. Samples were visualised with a Nikon A1R confocal laser scanning microscope with the NIS-Element software. The acquisition of signals from specific fluorophores was obtained by recording variable emission bandwidth with the virtual band mode. A 409-nm laser was used to detect chlorophyll autofluorescence from dinoflagellate cells, while the fluorophores Texas Red and AlexaFluor 568, 594 and 488 were specifically excited by the lasers reported in Table 1 . An oil immersion objective of $\times 60$ magnification and 1.4 numerical aperture was used to image three different sections of each well, hence 12 observations were made for each algal species and lectin treatment. The image of the algal species and lectin was unmixed against its negative control to remove any background signal caused by algal chlorophyll autofluorescence. A macro was developed to process $\mathrm{Nd} 2$ files using $\mathrm{Fiji}^{\mathrm{TM}}$ and obtain the mean fluorescence intensity (MFI) of the signal provided by the lectin binding to the symbiont surface. Twenty-five algal cells were randomly selected from each image, and hence 300 cells were analysed for each sample of a Symbiodiniaceae species incubated with each lectin. Data were tested for normality with the Shapiro-Wilk test and, when parametric assumptions were not met, we used the Kruskal-Wallis test to explore the fluorescence intensity profiles of different lectins binding to the surface of a Symbiodiniaceae species. Finally, the Tukey's test was used for multiple comparisons $(p>0.05)$.

Table 1. Fluorescent molecular probes conjugated with lectins used in this study for analysis of the Symbiodiniaceae cell surface at the confocal microscope.

\begin{tabular}{|llll|}
\hline Lectin probe & Glycan affinity & Fluorophore & Laser \\
\hline ConA & D-mannose; D-glucose & AlexaFluor 488 & 488 \\
\hline GS-II & $\begin{array}{l}\text { Terminal, non-reducing } \alpha-\text { I } \\
\beta \text {-N-acetyl-D-glucosaminyl }\end{array}$ & AlexaFluor 594 & 561 \\
\hline LPA & $\begin{array}{l}\text { N-acetylneuraminic acid; } \\
\text { glycuronic acid }\end{array}$ & FITC & 488 \\
\hline PhaL & N-acetyl-D-glucosamine & AlexaFluor 488 & 488 \\
\hline PNA & D-galactose & AlexaFluor 568 & 561 \\
\hline SBA & $\begin{array}{l}\text { N-acetyl-D- } \\
\text { galactosaminea }\end{array}$ & AlexaFluor 594 & 561 \\
\hline UEA & $\alpha$-L-Fucose & Atto 594 & 561 \\
\hline WGA & N-acetyl-D-glucosamine & Texas Red & 561 \\
\hline
\end{tabular}

ConA concanavalin A, GS-Il lectin from Griffonia simplicifolia, LPA lectin from Limulus polyphemus (horseshoe crab), PhaL lectin from Phaseolus vulgaris, PNA lectin from Arachis hypogaea (peanut), SBA lectin from Glycine max (soybean), UEA lectin from Ulex europaeus, WGA wheat germ agglutinin.
Lectin array of Symbiodiniaceae cell-surface glycoproteins

To isolate glycoproteins from the Symbiodiniaceae cell surface, we used guanidinium chloride (GuHCl) extraction [34]. We confirmed that the extraction of cell-surface glycoproteins/proteins did not result in lysis of the algal cell by using Calcofluor-white to stain the cellulosic algal cell wall [35]. Calcofluor-stained algae were visualised with a Nikon A1R confocal laser scanning microscope with a 405-nm laser to detect stained cellulose and a 409-nm laser to detect chlorophyll autofluorescence from dinoflagellate cells.

For each algal species, an aliquot of $1 \times 10^{8}$ cells $/ \mathrm{ml}$ was centrifuged at $1500 \times g$, washed twice in $1 \times \mathrm{PBS}$, resuspended in $3 \mathrm{ml}$ of $6-\mathrm{M} \mathrm{GuHCl}$ and divided into two tubes. After $4 \mathrm{~h}$ of incubation at RT under constant shaking at $800 \mathrm{rpm}$, cells were pelleted at $15,000 \times g$ for $30 \mathrm{~min}$ at RT and discarded; the supernatant of the two tubes was then concentrated and combined with an Amicon Ultra MWCO 3-KDa filter, and the protein concentrations of the samples were checked with the Pierce BCA Protein Assay. Each algal species had $n=4$ replicates. Samples were run on a Ray Biotech Lectin array 95, which has 95 different lectins (Supplementary information 1). One milligram per millilitre of glycoprotein suspension was run on the lectin array by Crux Biolabs Australia. The binding of glycoproteins to different lectins produced fluorescence intensity signals informative of a certain glycan abundance. The data were backgroundcorrected, tested for normality and a one-way Anova used to explore the binding of a certain lectin among Symbiodiniaceae cell-surface glycoproteins. Finally, the Tukey's test was used for multiple comparisons $(p>0.05)$, and the variation in B. minutum, C. goreaui and $F$. kawagutii cell-surface glycomes was visualised using principal component analysis (PCA).

\section{Cell-surface alteration and host-symbiont inoculation experiments}

A total of 1053 anemones (oral disc diameter of $\sim 5 \mathrm{~mm}$ ) was chemically bleached of algal symbionts as previously described [36]. After bleaching, anemones were kept in RSS and fed with brine shrimp. One week prior to inoculation with Symbiodiniaceae symbionts, feeding was discontinued, and anemones were transferred to 12-well plates such that each 12-well plate contained $n=3$ anemone genotype replicates, organized randomly to minimize well effect. Each 12-well plate had $n=3$ technical replicates, all altered with the same enzymatic or masking treatment and inoculated with one symbiont type.

The anemone surface lectins were masked with the sugars D-glucose, Dmannose, D-galactose, methyl- $\alpha$ - and $\beta$-D-galactose, L-fucose, D-xylose, Dgalacturonic acid, D-glucuronic acid and L-rhamnose, all at a concentration of $10 \mathrm{mM}$ (Table 2; [20]). Organisms were incubated in RSS supplemented with one of the sugars for an hour under the conditions described above, and subsequently inoculated with one of the three Symbiodiniaceae species. Control organisms did not receive any sugar prior to symbiont inoculation.

The symbiont cell surface was altered via digestion with one of the two enzymes: a-amylase [29] or trypsin [18, 26, 29, 30]. Enzyme specificities are reported in Table 2. Algal cells were sampled in the exponential growth

Table 2. Enzymes used to alter Symbiodiniaceae cell-surface molecules and carbohydrates used to mask E. diaphana cell-surface lectins, prior to inoculation experiments.

\begin{tabular}{|c|c|c|c|c|}
\hline Organism & Treatment & Product \# & Concentration & Enzyme specificity \\
\hline Symbiodiniaceae & $\alpha$-amylase & A6255 & $5 \mathrm{mg} / \mathrm{ml}$ & $\alpha-(1,4)$ glycan linkages \\
\hline Symbiodiniaceae & Trypsin & T6567 & $6 \mu \mathrm{g} / \mathrm{ml}$ & Peptides on the C-terminal side of lysine and arginine residues \\
\hline E. diaphana & D-galactose & G0750 & $10 \mathrm{mM}$ & I \\
\hline E. diaphana & Methyl- $\beta$-D-galactose & G1125 & $10 \mathrm{mM}$ & I \\
\hline E. diaphana & D-glucose & 47829 & $10 \mathrm{mM}$ & / \\
\hline E. diaphana & D-glucuronic acid & G5269 & $10 \mathrm{mM}$ & / \\
\hline E. diaphana & L-fucose & F2252 & $10 \mathrm{mM}$ & / \\
\hline E. diaphana & L-rhamnose & W373011 & $10 \mathrm{mM}$ & I \\
\hline E. diaphana & D-xylose & PHR2102 & $10 \mathrm{mM}$ & / \\
\hline
\end{tabular}

All products were purchased from Sigma-Aldrich. 
phase, pelleted and resuspended in a digestion solution composed of RSS and one of the enzymes used in the study. After a 2-h digestion step at $27^{\circ} \mathrm{C}$ in the dark in a shaking incubator at $90 \mathrm{rpm}$, the symbionts were washed twice in RSS and used immediately to inoculate the bleached, unmodified anemones. Control organisms received untreated symbionts.

For both host and symbiont surface modification experiments, inoculations were performed as previously described [27]. Given that most Symbiodiniaceae cell-surface glycans recover within $48-72 \mathrm{~h}$ postenzymatic cleaving [30], anemones were anesthetized with $\mathrm{MgCl}_{2} 2$ days post-inoculation, and tentacles then sampled, fixed and microscopically analysed to assess symbiont cell densities in E. diaphana [27]. Data were log-transformed to achieve normality and a generalized linear model (GLM) with a Gaussian distribution was used to test the influence of $E$. diaphana genotype (i.e. AIMS2, 3 and 4), Symbiodiniaceae species (i.e. $B$. minutum, C. goreaui and F. kawagutii) and treatment (i.e. enzymatic treatment of symbiont surface or masking of host lectins with carbohydrates) on symbiont uptake by the host (as number of symbiont cells $/ \mathrm{mm}^{2}$ in anemone tentacles). Analyses were conducted in R v. 3.6.1 [37] with the package Tidyverse [38]. The GLM specification for the model was:

\section{$\operatorname{glm}(\boldsymbol{N} \sim$ host genotype $*$ symbiont species $*$ treatment, family $=$} gaussian $(\operatorname{link}=\log ))$

Best model selection was performed by comparing the full model with all the effects and interactions against the model without each of the effects or interactions and confirmed using the Akaike Information Criterion (AIC, 15395.97). Analysis of variance was used to test the significance of the overall fixed effects fitted in the model.

\section{RESULTS \\ Nucleotide sugar and monosaccharide composition analysis of Symbiodiniaceae cell walls}

The surface of the symbiont cell constitutes an intricate mesh of cellulose, glycoproteins and polysaccharides [17]. In eukaryotes, matrix polysaccharides are synthesized from activated sugar substrates called nucleotide sugars, most of which are transferred by nucleotide sugar transporters from the cytosol into the Golgi apparatus where they are assembled into glycan polymers by various glycosyltransferases, and then transported via vesicles to the cell wall [31]. Therefore, we decided to investigate the nucleotide sugar profiles from whole cells to gain insight into possible end products present in the dinoflagellate wall. We detected various nucleotide sugars in the three species of Symbiodiniaceae investigated (Fig. 1). The respective sugar residues can be present in cell-wall polymers, glycoproteins and/ or surface glycans.

We then examined the monosaccharide composition of insoluble cell-wall extracts from each of the three Symbiodiniaceae species (Fig. 2). All cell-wall monosaccharides identified were also evident in their respective precursor form in the nucleotide sugar analysis (Fig. 1). The most abundant monosaccharide detected in the three species was glucose (Fig. 2). D-mannose was the only monosaccharide for which the relative amount did not statistically differ among the three Symbiodiniaceae species ( $p>0.05$; Fig. 2). The relative amounts of L-fucose, L-rhamnose, Dgalactose, D-glucose, D-xylose and D-galacturonic acid differed among species $(p<0.05$; Fig. 2$)$, while D-glucuronic acid was dissimilar between $B$. minutum and $F$. kawagutii only $(p<0.05$; Fig. 2). L-arabinose, $\mathrm{N}$-acetyl-galactosamine and -glucosamine were present in trace amounts. We therefore did not further investigate the effect of these monosaccharides on symbiosis establishment.

\section{Confocal microscopy of Symbiodiniaceae cell surfaces}

The MFI profile of different lectin conjugates varied among the three Symbiodiniaceae species (Fig. 3). The lectins ConA, GS-II, LPA, PhaL, SBA, UEA and WGA bound significantly to the surface of $B$. minutum. The $C$. goreaui surface was populated by glycans with an affinity to ConA, GS-II, LPA, PhaL, PNA, SBA and UEA. The surface of $F$. kawagutii exhibited significant binding for all the lectin conjugates used in the study. ConA, which specifically binds to terminal D-mannose and D-glucose residues, had a stronger intensity profile in C. goreaui compared with $B$. minutum and $F$. kawagutii $(p<0.001)$. The latter two species showed similar binding of the probe to their cell surfaces. The lectins GS-II, LPA, PhaL, SBA and UEA all bound to B. minutum, C. goreaui and $F$. kawagutii, and showed different MIFs among symbiont species. C. goreaui and F. kawagutii presented different amounts of D-galactose on their cell surfaces, as revealed with the lectin probe PNA $(p<0.001)$; however, the lectin WGA identified a dissimilar abundance of glycoconjugates containing $\mathrm{N}$-acetyl-D-glucosamine residues on $B$. minutum and $F$. kawagutii $(p<0.001)$.

\section{Lectin array of Symbiodiniaceae cell-surface glycoproteins}

Confocal microscopy showed an intact Calcofluor-stained cell wall surrounding the Symbiodiniaceae cells after the $\mathrm{GuHCl}$ treatment (Supplementary information 2), which we interpret as the extraction procedure not disrupting the majority of cells.

Fifty-four percent of the lectins showed a difference in their affinity to the three Symbiodiniaceae species (Supplementary information 1 and 3). In line with the confocal microscopy results, the Symbiodiniaceae surface glycome varied among B. minutum, C. goreaui and F. kawagutii, and PCA showed that cell-surface glycoproteins clustered according to symbiont species (Fig. 4). The presence/absence of D-mannose, D-glucose, D-galactose, Lfucose, N-acetyl-D-galactosamine and $\mathrm{N}$-acetyl-D-glucosamine identified via confocal microscopy was confirmed by the lectin array, but the array did not detect any statistically supported difference among algal species for the lectins PhaL, SBA, UEA and WGA (Supplementary information 1 and 3). This discrepancy may be attributed to the sensitivity of each method, as we discuss later in this work.

Lectins of the array that were specific for NANA showed a light binding to the glycoproteins of the three algal cells (Supplementary information 3), as also shown by the binding of the LPA lectin probe (Fig. 3). Sialic acids, usually characteristics of higher invertebrates, have been found composing a small percentage of the Symbiodiniaceae N-glycome [24]. Future directions should explore the role of these molecules in the algal cell.

\section{Cell-surface alteration and host-symbiont inoculation experiments}

The density of $B$. minutum and $C$. goreaui populating E. diaphana at $48 \mathrm{hpi}$ varied considerably (Fig. 5 and Table 3). Congruent with findings in our previous study [27], E. diaphana genotype influenced the start of symbiosis (GLM, df $=2, p<0.001$; Supplementary information 4), although a clear pattern between host identity and symbiosis establishment was not found. Symbiont species (GLM, df $=1, p<0.001)$, treatment $(\mathrm{GLM}, \mathrm{df}=$ $12, p<0.001)$ and the interaction of the three factors (GLM, df $=$ 24, $p<0.001)$ significantly affected the establishment of new associations. The homologous species ( $B$. minutum) colonised the anemones rapidly, whereas the compatible heterologous species (C. goreaui) also colonised the animals, but at a lower density, and the incompatible strain ( $F$. kawagutii) did not measurably colonise the hosts (Fig. 5 and Table 3).

After exposing aposymbiotic anemones to certain monosaccharides, we observed significantly lower in hospite $B$. minutum and C. goreaui cell densities at 48 hpi for D-galactose $\left(p_{B}<0.001\right.$; $\left.p_{C}<0.05\right)$, L-fucose and D-xylose $\left(p_{B}<0.001 ; p_{C}<0.05\right)$. Incubation of the aposymbiotic hosts with D-glucose, D-mannose or Dgalacturonic acid resulted in lower algal densities (compared to the controls) for $B$. minutum, but revealed no difference for $C$. goreaui $\left(p_{B}<0.001 ; p_{C}>0.05\right)$. Exposing the hosts to a-D-galactose 


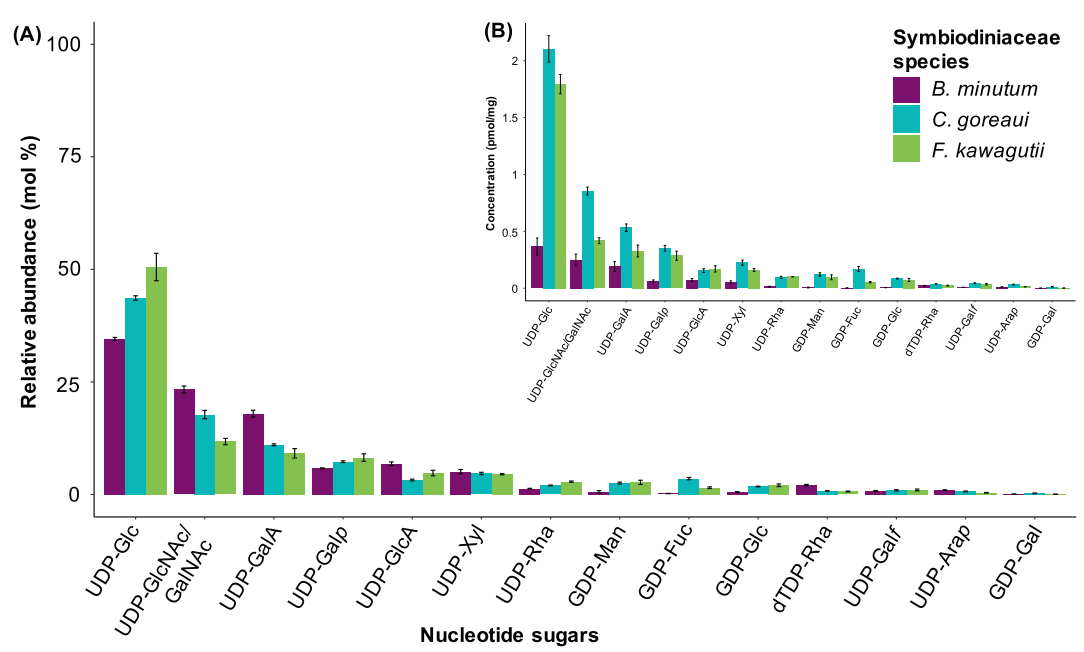

Fig. 1 Nucleotide sugars present in B. minutum (purple), C. goreaui (blue) and F. kawagutii (green). A Each bar represents the relative abundance (mean molar percentage $\pm \mathrm{SEM}$ ) of a nucleotide sugar in the algal cell. $\mathbf{B}$ Concentration (pmol/mg $\pm \mathrm{SEM}$ ) of a nucleotide sugar in the algal cell. UDP-Glc = UDP-glucose, UDP-GlcNAc/GalNAc = UDP-N-acetylglucosamine and UDP-N-acetylgalactosamine, UDP-GalA = UDPD-galacturonic acid, UDP-Gal = UDP-galactose, PAPS = adenosine 3'-phosphate 5'-phosphosulfate, UDP-Xyl = UDP-xylose, UDP-GIcA = UDPD-glucuronic acid, GDP-Fuc = GDP-fucose, GDP-Man = GDP-mannose, UDP-Rha = UDP-rhamnose, GDP-Glc $=$ GDP-D-glucose, UDP-Galf $=$ UDP-galactofuranose, dTDP-Rha = dTDP-rhamnose, UDP-Arap = UDP-arabinopyranose, GDP-Gal = GDP-galactose.

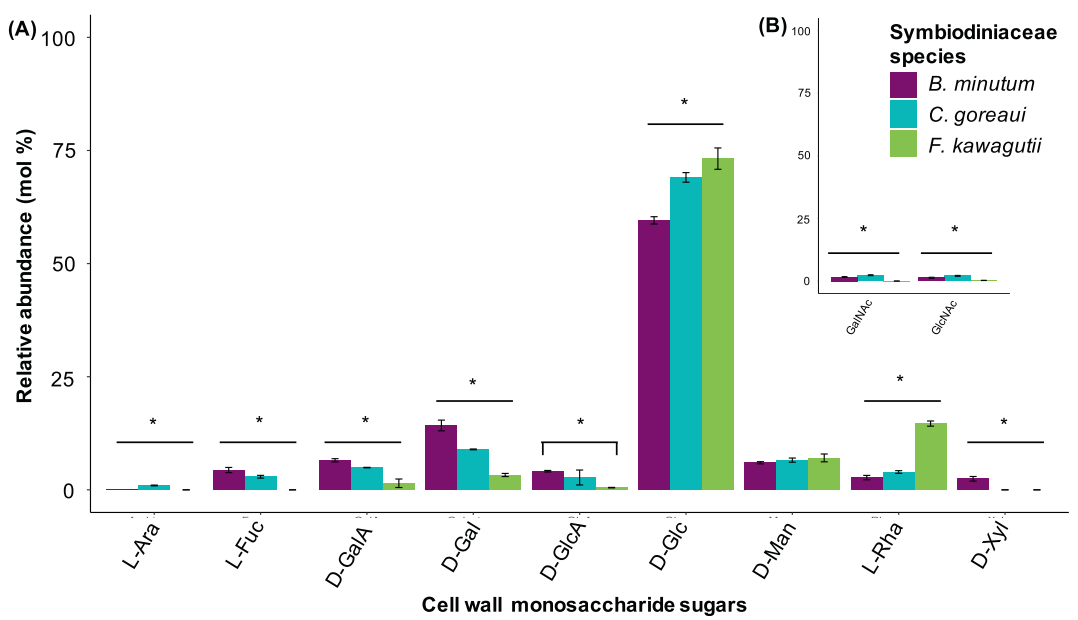

Fig. 2 Monosaccharide composition of hydrolysed alcohol insoluble residues extracted from the cell wall of $B$. minutum (purple), $C$. goreaui (blue) and F. kawagutii (green). A Monosaccharide analysis first isocratic elution step was performed with $4 \mathrm{mM}$ or B with $8 \mathrm{mM}$. Each bar represents the relative abundance (mean molar percentage \pm SEM) of a monosaccharide. Stars indicate statistically supported differences. L-Ara $=\mathrm{L}$-arabinose, $\mathrm{L}$-Fuc $=\mathrm{L}$-fucose, $\mathrm{D}$-GalA $=\mathrm{D}$-galacturonic acid, D-Gal $=\mathrm{D}$-galactose, D-GlcA $=\mathrm{D}$-glucuronic acid, D-Glc $=\mathrm{D}$ glucose, $\mathrm{D}-\mathrm{Man}=\mathrm{D}$-mannose, L-Rha $=$ L-rhamnose, $\mathrm{D}-\mathrm{Xyl}=\mathrm{D}$-xylose, GalNAc $=\mathrm{N}$-acetylgalactosamine, GlcNAc $=\mathrm{N}$-acetylglucosamine.

and L-rhamnose had no effect on symbiosis establishment with any of the two compatible symbionts $(p>0.05)$, whereas the presence of $\beta$-D-galactose resulted in a super-infection for both $B$. minutum and $C$. goreaui $(p<0.001)$. The inability of $F$. kawagutii to colonise E. diaphana did not change $(p>0.05)$ as a result of any of the monosaccharide host-masking trials (Fig. 5 and Table 3). Incubation of E. diaphana with D-glucuronic acid was lethal to the anemones, hence it was not possible to collect data for this treatment.

Digesting proteins at the symbiont surface with the enzyme trypsin prior to inoculation significantly affected the uptake of both compatible symbionts $\left(p_{B}<0.001 ; p_{C}<0.05\right)$, severely reducing the success of $B$. minutum but marginally increasing $C$. goreaui cell density (Fig. 5 and Table 3). Alpha-amylase had no influence on the density of either $B$. minutum or $C$. goreaui found in the host compared to the control ( $p>0.05$; Fig. 5 and Table 3 ). The infectivity of $F$. kawagutii for all genotypes of $E$. diaphana did not differ significantly $(p>0.05)$ from the control after a-amylase or trypsin pre-digestion (Fig. 5 and Table 3).

\section{DISCUSSION}

We explored the involvement of glycan-lectin interactions in the establishment of endosymbiosis between the sea anemone, $E$. diaphana and three species of Symbiodiniaceae algae, the homologous $B$. minutum, the heterologous-compatible $C$. goreaui and the heterologous-incompatible $F$. kawagutii.

As expected, we found good concordance between the nucleotide sugar repertoire in the three Symbiodiniaceae species and our monosaccharide analysis, which is consistent with these precursors finding their way into glycan structures such as those making up the cell walls. The cell-surface glycome varies among $B$. minutum, $C$. goreaui and $F$. kawagutii, as shown by the monosaccharide analysis (Fig. 2), confocal microscopy 

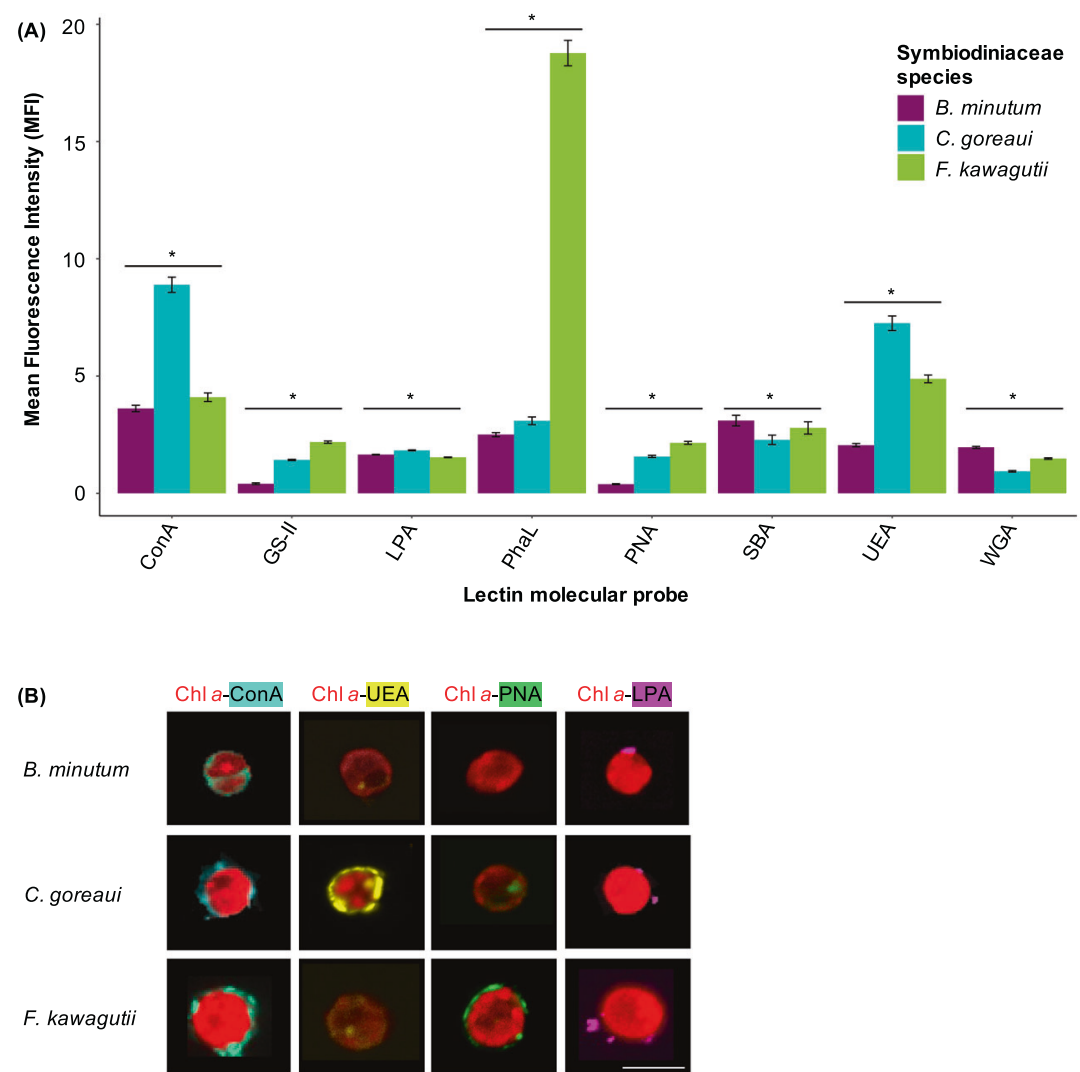

Fig. 3 Confocal microscopy of B. minutum (purple), C. goreaui (blue) and F. kawagutii (green) cell surfaces. A Mean fluorescence intensity $(\mathrm{MFI} \pm \mathrm{SEM})$ of lectin conjugate binding to the cell surface of a Symbiodiniaceae species. ConA = concanavalin A, GS-II = lectin from Griffonia simplicifolia, LPA = lectin from Limulus polyphemus (horseshoe crab), PhaL = lectin from Phaseolus vulgaris, PNA = lectin from Arachis hypogaea (peanut), SBA = lectin from Glycine max (soybean), UEA = lectin from Ulex europaeus, WGA = wheat germ agglutinin. Stars indicate statistically supported differences. B Representative cells of B. minutum, C. goreaui and F. kawagutii labelled with the lectin probes ConA (concavalin A specific for D-mannose and D-glucose; light blue), UEA (lectin from Ulex europaeus specific for $\alpha$-L-fucose; yellow), PNA (lectin from Arachis hypogaea specific for lactose and D-galactose; green) and LPA (lectin from Limulus polyphemus specific for N-acetylneuraminic acid and glucuronic acid; magenta) and imaged using confocal microscopy. Scale $=10 \mu \mathrm{m}$.

(Fig. 3) and the lectin array results (Fig. 4 and Supplementary information 3).

\section{Glucose and mannose}

D-glucose stands out as highly abundant in both the nucleotide sugar pools and in the cell-wall extracts of the three Symbiodiniaceae species (Figs. 1 and 2). The symbiont cell wall is predominantly composed of cellulose [17], a polysaccharide consisting of $\beta$-linked D-glucose, so the abundance of this molecule across the three algal species is not surprising. Binding of the lectin molecular probe ConA, which is specific for D-glucose (and D-mannose), to all the three algal species (Fig. 3) is also consistent with cellulose in the algal cell walls. B. minutum, C. goreaui and F. kawagutii cell-surface glycoproteins bound to several lectins specific for glucose and mannose in the lectin array study (i.e. LCHA and PSA; Supplementary information 1 and 3). When we treated the anemones with D-glucose prior to inoculation with symbionts, it significantly decreased the colonisation success of the homologous $B$. minutum but had no impact on the colonisation success of C. goreaui and F. kawagutii (Fig. 5 and Table 3).

D-mannose was the only sugar that did not differ significantly in mole percentage amongst the three species of Symbiodiniaceae (Fig. 2). Previous work has shown that high-mannose glycans constitute $52 \%$ of the B. minutum cell-surface N-glycome [24], and the most abundant protein on the cell surface of symbiotic dinoflagellates found by Lin and co-workers bears a terminal mannose residue [18]. Here, lectins specific for high mannose such as BC2L-A, CALSEPA, GRFT and ORYSATA recognised glycoproteins of the three Symbiodiniaceae species in the array experiment (Supplementary information 1 and 3). Furthermore, two mannosebinding lectins, 'Millectin' from Acropora millepora [19] and 'PdC lectin' from Pocillopora damicornis [39], bind around the algae in the gastrodermis of the host [39] and are characterised by extensive sequence variation of the binding region, which may reflect the capacity to recognise both beneficial symbionts and pathogens [19]. Here, treating the anemones with D-mannose prior to inoculation with symbionts decreased the colonisation success of the homologous $B$. minutum but had no effect on experiments with C. goreaui and F. kawagutii (Fig. 5 and Table 3). ConA negatively influenced symbiosis establishment between the coral Fungia scutaria and its symbionts [26], but, in the case of $A$. tenuis, the lectin had no effect on symbiotic colonisation [29]. Taken together, these results suggest that both D-mannose and Dglucose residues are likely important functional components of the symbiont surface that participate in recognition of Symbiodiniaceae and maintenance of the mutualism, rather than just being crucial monosaccharides responsible for the discrimination between compatible and incompatible symbionts.

\section{D-galacturonic acid}

The relative amount of D-galacturonic acid was different among Symbiodiniaceae species, being more abundant in the compatibles B. minutum and $C$. goreaui compared to the non-compatible $F$. kawagutii (Fig. 2). When we incubated the anemones in Dgalacturonic acid, colonisation success remained unaltered for $F$. 
kawagutii and decreased for both B. minutum and C. goreaui, although the decrease was only statistically supported for $B$. minutum (Fig. 5 and Table 3). We speculate that D-galacturonic acid may be a component of the glycoproteins involved in the recognition of the compatible symbionts.

\section{D-galactose}

The presence of D-galactose molecules on the symbiont cell wall (Fig. 2) was confirmed via confocal microscopy with the lectin probe PNA (Fig. 3), and with the binding of algal surface glycoproteins to galactose-specific lectins on the array, such as MNAG, RCA120 and RCA60 (Supplementary information 1 and 3). Interestingly, monosaccharide analysis showed D-galactose to be more abundant in or on the cell wall of the homologous $B$. minutum compared to the heterologous $C$. goreaui and the incompatible F. kawagutii (Fig. 2). Previous studies reported the presence of D-galactose on the surface of several Symbiodiniaceae species [24, 25], and the colonisation of $A$. tenuis larvae during symbiosis establishment was negatively influenced by the presence of the sugar [20].

Here, D-galactose significantly decreased colonisation success of the homologous $B$. minutum and the heterologous-compatible C. goreaui in E. diaphana, compared to the control (Fig. 5 and Table 3). Thus, several lines of evidence support the involvement of D-galactose in the establishment of symbiosis between

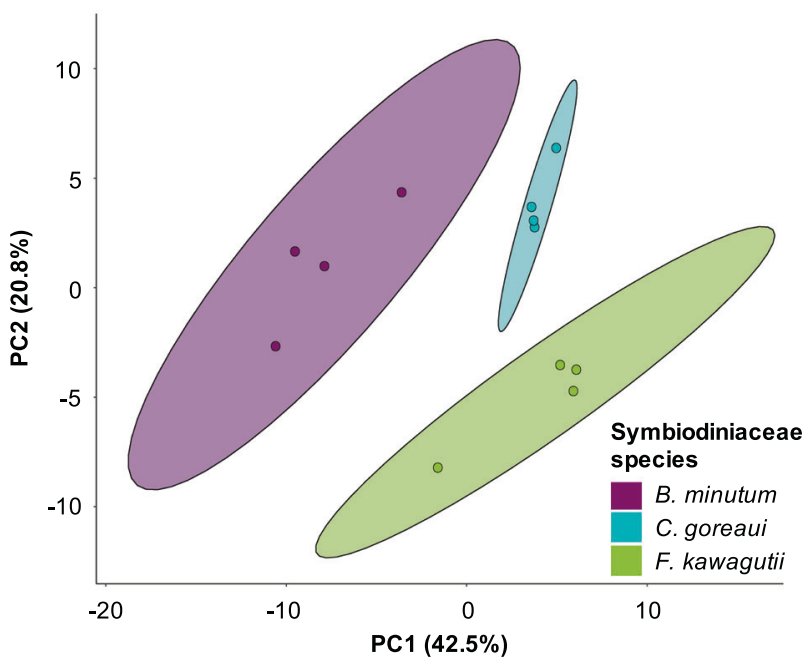

Fig. 4 Affinity of B. minutum (purple), C. goreaui (blue) and $F$. kawagutii (green) cell-surface glycoproteins for lectins. PCA visualization of Symbiodiniaceae glycoconjugates binding to 95 lectins on the array. cnidarians and Symbiodiniaceae. For instance, the D-galactosebinding lectin SLL-2 has been isolated from the coral Sinularia lochmodes. This protein was localised surrounding the symbiont cells when resident in the gastrodermis of the host, and in vitro experiments showed the ability of SLL-2 to arrest the dinoflagellate in the typical symbiotic state, viz non-dividing and nonmotile [21, 40, 41, 42]. Another lectin (CeCL) that binds to carbohydrate chains with a D-galactosyl moiety was found in Fungia echinate, and-similar to SLL-2-CeCL transformed Symbiodiniaceae cells from a flagellated and motile state to a coccoid and non-motile form [22].

Considering that the activity of $\mathrm{CeCL}$ on the symbiont cells was concentration-dependent [22], and that in our study Dgalactose was found to be more abundant in the compatible symbionts than the incompatible one, this raises the question whether the relative amount of a certain glycan has an impact on symbiont selection. Another open question is whether the stereochemistry of a sugar is important in symbiont selection. To further explore the possible role of D-galactose in symbiont selection, we utilised different conformations of the molecule, namely methyl- $\alpha$ - or methyl- $\beta-D$-galactose, which are fixed isomers due to the methyl group. Intriguingly, the methyl-asugar had no effect on host colonisation, whereas methyl- $\beta$-Dgalactose significantly altered the establishment of symbiosis between the host and compatible symbionts, by reducing the number of $B$. minutum but increasing the number of $C$. goreaui present in the host at $48 \mathrm{hpi}$ (Fig. 5 and Table 3). We conclude that D-galactose, in particular D-galactose when it is $\beta$-linked rather than $a$-linked, is a crucial player of the lock-and-key mechanism responsible for recognising suitable symbionts during the establishment of symbiosis.

\section{Fucose and xylose}

Like D-galactose, L-fucose and D-xylose are more abundant in extracts from the homologous $B$. minutum than the heterologous C. goreaui and F. kawagutii (Fig. 2), and the lectin probe UEA confirmed the presence of L-fucose exposed on the surface of symbiont cells (Fig. 3). Accordingly, PA-IIL and RSFUC, specific for fucose, bound to the surface glycome of the three algal species in the lectin array (Supplementary information 1 and 3). Both carbohydrates have been associated with Symbiodiniaceae before, as N-glycan glycosyltransferases for fucose (fucosyltransferases) and xylose (xylosyltransferases) are encoded in the algal genome [24]. Here, we show that both L-fucose and D-xylose decrease the colonisation success of $B$. minutum and C. goreaui in E. diaphana (Fig. 5 and Table 3). Although the role of these sugar residues at the initiation of symbiosis needs further exploration, these results suggest a function in the recognition of compatible algal types.

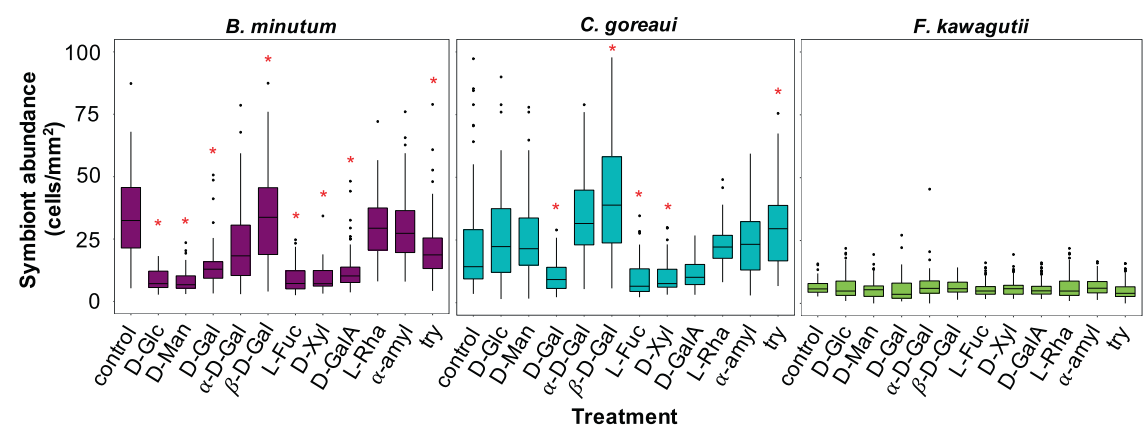

Fig. 5 Cell surface alterations and inoculation experiments of the host with B. minutum (purple), C. goreaui (blue) and $F$. kawagutii (green). Symbiont abundance (cells $/ \mathrm{mm}^{2}$ ) in the tentacles of $E$. diaphana, $48 \mathrm{~h}$ post-inoculation after masking of the host surface lectins with the carbohydrates D-Glu = D-glucose, D-Man = D-mannose, D-Gal = D-galactose, $\alpha$-D-Gal = methyl-alpha-D-galactose, $\beta$-D-Gal $=$ methylbeta-D-galactose, L-Fuc = L-fucose, D-Xyl = D-xylose, D-GalA = D-galacturonic acid, L-Rha $=$ L-rhamnose or enzymatic treatment of the Symbiodiniaceae surface with $\alpha$-amyl $=\alpha$-amylase or try $=$ trypsin, and compared to the control. Red stars indicate statistically supported differences compared to control. 
Table 3. Effect of treatments (host lectin masking and symbiont cell-surface enzymatic modification) on the colonization of $E$. diaphana by $B$. minutum, C. goreaui and F. kawagutii.

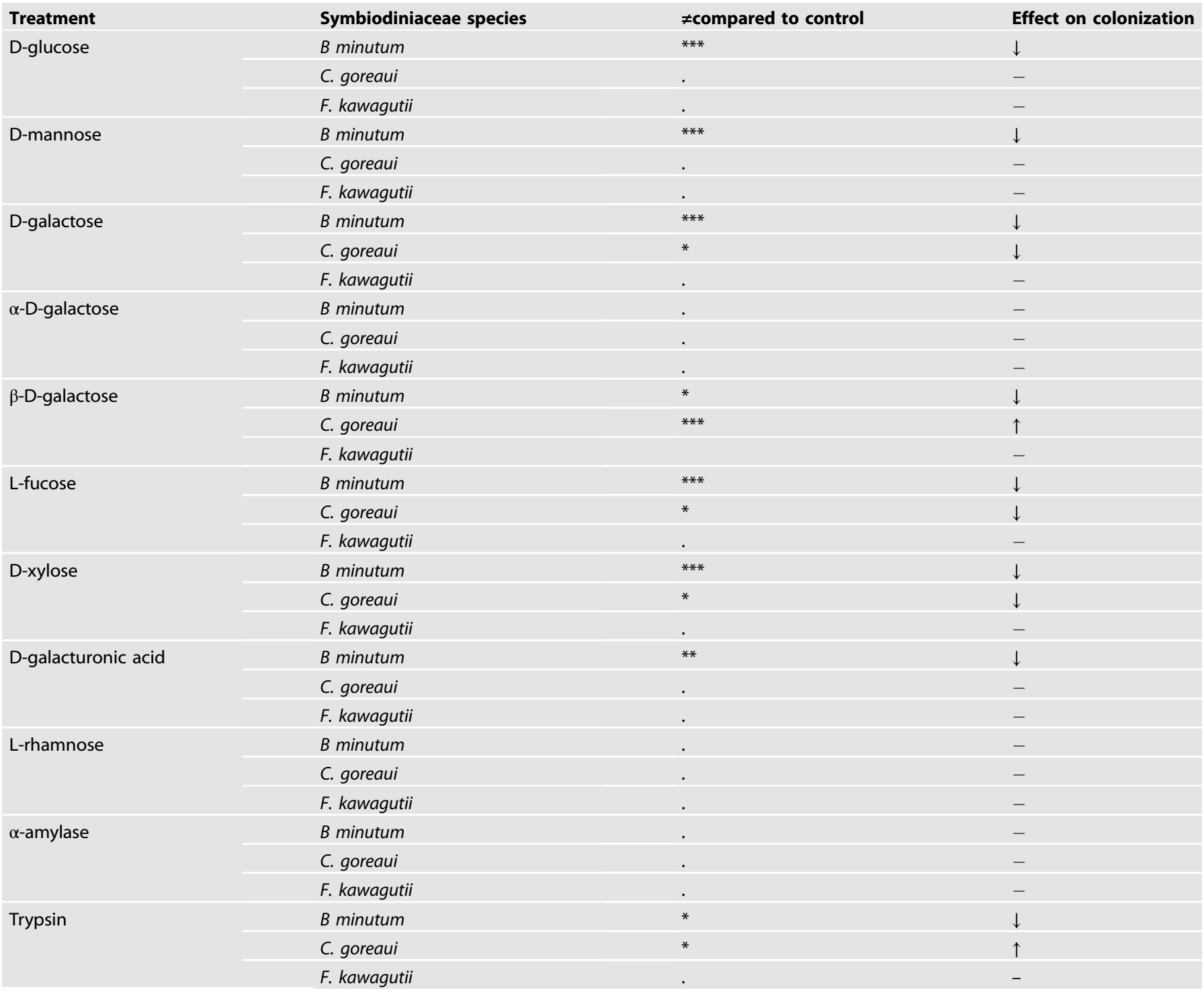

Stars indicate statistically supported differences of the number of cells $/ \mathrm{mm}^{2}$ of a Symbiodiniaceae species compared to the control. . $=p>0.05$. $(\downarrow)$ means the treatment decreased re-infection of the anemones by the symbiont; (-) means the treatment had no influence and allowed re-infection of the anemones by the symbiont; $(\uparrow)$ means the treatment resulted in a super-infection of the anemones by the symbiont.

${ }^{*} p<0.05 ;{ }^{* *} p<0.01 ;{ }^{* *} p<0.001$.

\section{N-acetyl hexosamine sugars}

Our HPAEC-PAD analysis detected only small traces of $\mathrm{N}$ acetylglucosamine and $\mathrm{N}$-acetylgalactosamine among the cellwall monosaccharides of B. minutum, C. goreaui and F. kawagutii $(<1.5 \mathrm{~mol} \%)$. Nevertheless, cell-surface glycoproteins of the three algal species bound several $\mathrm{N}$-acetyl groups-specific lectins (i.e. GS-II, PhaL, SBA and WGA), either in the fluorescence microscopy assay or lectin array or both. Although Logan and co-workers showed identical binding of these probes to various Symbiodiniaceae species [25], our confocal microscopy study found that they bind differently to the surface of our strains of $B$. minutum, $C$. goreaui and F. kawagutii from the GBR (Fig. 3). In particular, the incompatible $F$. kawagutii presented high levels of $\mathrm{N}$-acetyl-Dglucosamine compared to the compatibles $B$. minutum and $C$. goreaui (Fig. 3). In a previous study, two N-acetyl-D-galactosamine binding lectins were isolated from the scleractinian $A$. tenuis, and seem to be involved in the acquisition of Symbiodiniaceae of the Symbiodinium and Durusdinium genera [20]. In another study, carbohydrates with $\mathrm{N}$-acetylglucosamine residues decreased the acquisition of compatible symbionts in $A$. tenuis. The N-acetyl-Dglucosamine-binding lectin ActL attracted compatible Symbiodiniaceae to the coral host, suggesting a role in chemotactic attraction prior to recognition and uptake of the symbiont to start symbiosis [43]. Perhaps, $\mathrm{N}$-acetyl residues characterize symbionts incompatible with our host, $E$. diaphana from the GBR. The role of $\mathrm{N}$-acetylated sugars remains to be determined, and further experiments are needed to uncover the role of these molecules in the cnidarians-Symbiodiniaceae symbiosis.

\section{Large and a-linked polysaccharides, and L-rhamnose}

Treating the three symbiont species with a-amylase or challenging them against $E$. diaphana in the presence of L-rhamnose, did not 
alter the ability of the symbionts to colonize E. diaphana (Fig. 5 and Table 3). Starch-like structures are not typical components of dinoflagellate cell walls, so it is not surprising that a-amylase treatment had no effect on establishment of symbiosis here and in a previous study [26]. Indeed, a-linked polysaccharides and Lrhamnose do not seem to be crucial in establishment of the cnidarian-dinoflagellate symbiosis.

\section{Trypsin treatment}

Trypsin is a protease that cleaves peptide bonds on the C-terminal side of lysine and arginine amino acid residues. It has been used widely on Symbiodiniaceae $[18,26,30]$ to examine the contribution of symbiont cell wall or surface protein moieties to host cell invasion. Previous studies have shown that homologous symbiont colonisation success was negatively affected by trypsin treatment in E. diaphana from Taiwan [18] and larvae of the coral F. scutaria [26]. Conversely, an experiment on $A$. tenuis larvae in which glycoconjugates of algal surface glycoproteins were digested with trypsin resulted in a super-infection by $C$. goreaui, one of the compatible symbionts during the larval stage of this coral species [29]. In our study, trypsin treatment negatively influenced colonisation by the homologous $B$. minutum but enhanced uptake of $C$. goreaui, resulting in a super-infection of the anemones (Fig. 5 and Table 3). A specific configuration of glycoproteins might, on one hand, ensure the recognition of $C$. goreaui as a compatible partner; on the other hand, it might be the crucial factor that regulates the uptake of a symbiont that, although compatible, is not the homologous type of $E$. diaphana from the GBR. The alteration of these glycan structures, besides altering the establishment of symbiosis with compatible symbionts, might also allow $C$. goreaui to more easily invade the host by avoiding the host mechanism of minimizing the uptake of heterologous algae.

\section{Concordances and differences between the glycome analytical methods}

Our multi-technology approach to characterize the glycomes of differently compatible symbionts is the first of its kind. A high degree of concordance between the results obtained from HPAEC$P A D$, confocal and lectin array analyses emphasizes the utility of using multiple assays to explore the glycomes. Nevertheless, some inconsistencies between the analyses were evident. The three methods rely on very different methodologies. For the HPAECPAD analysis, the Symbiodiniaceae cells were lysed, AIR was extracted, hydrolysed, concentrated and analysed. For confocal microscopy, the Symbiodiniaceae cells were fixed, incubated with labelled lectins, which were then visualized by fluorescence microscopy. For lectin array analysis, the Symbiodiniaceae cellsurface glycoproteins were extracted, concentrated, purified, biotin labelled and then exposed to the array of immobilized lectins. Thus, three of the methods aim to extract the glycans and then modify them for identification by different means whereas the microscopy approach sends lectins into the cell surface hoping that the glycan target is accessible. It is not surprising that some inconsistencies between sugars identified are apparent, but the overall concordance gives confidence in these approaches. Furthermore, our lectin array -in which we used $\mathrm{GuHCl}$ extraction to release glycoproteins-showed marked differences among different symbiont species; differences not reported in a previous lectin array analysis using a different extraction method [30]. Detailed inventories of the surface glycomes of compatible/ incompatible symbionts will help unravel which glycans are crucial to successful symbiosis.

\section{CONCLUSION}

The present study explored glycan-lectin interactions during the establishment of symbiosis between cnidarians and three species of Symbiodiniaceae, each with a different compatibility with the model symbiotic cnidarian E. diaphana. We adopted a multi-step approach and profiled cell-surface molecules via nucleotide sugar analysis, monosaccharide composition analysis, confocal microscopy and lectin array analysis. Once we had assessed the differences between the three cell-surface glycomes, we examined the importance of selected sugar residues in the initiation of a mutualistic relationship between the three algae and three genotypes of aposymbiotic $E$. diaphana from the GBR. D-glucose and D-mannose were the most abundant monosaccharides of $B$. minutum, C. goreaui and F. kawagutii. For these sugars, we envision a role as pivotal functional components of the symbiont surface glycome, which are probably involved in the recognition between beneficial or detrimental symbionts but not necessarily associated with host discrimination among Symbiodiniaceae species. Masking of host cell-surface lectins with L-rhamnose and the alteration of the symbiont surface with a-amylase had no influence on the establishment of symbiosis, and we therefore assume that these sugar residues are not critical. Sialic acids and $\mathrm{N}$-acetylgalactosamine showed an unclear pattern in the present analysis, which deserves further attention. Treatment of the algal cells with trypsin changed their rate of uptake, reinforcing previous conclusions that a protein-based moiety, likely a glycoprotein, is an essential part of compatible symbiont recognition. Intriguingly, our data also strongly support the importance of D-galactose and, in particular, $\beta$-D-galactose residues in the establishment of the cnidarian-dinoflagellate symbiosis, and we propose a potential involvement of other sugar residues such as L-fucose, D-xylose and D-galacturonic acid in the early steps of this mutualism.

\section{REFERENCES}

1. LaJeunesse TC, Parkinson JE, Gabrielson PW, Jeong HJ, Reimer JD, Voolstra CR, et al. Systematic revision of Symbiodiniaceae highlights the antiquity and diversity of coral endosymbionts. Curr Biol. 2018;28:2570-80.

2. Davy SK, Allemand D, Weis VM. Cell biology of cnidarian-dinoflagellate symbiosis. Microbiol Mol Biol Rev. 2012;76:229-61.

3. Nitschke MR, Craveiro SC, Brandão C, Fidalgo C, Serôdio J, Calado AJ, et al. Description of Freudenthalidium gen. nov. and Halluxium gen. nov. to formally recognize clades $\mathrm{Fr} 3$ and $\mathrm{H}$ as genera in the family Symbiodiniaceae (Dinophyceae). J Phycol. 2020;56:923-40.

4. Suggett DJ, Warner ME, Leggat W. Symbiotic dinoflagellate functional diversity mediates corals survival under ecological crisis. Trends Ecol Evol. 2017;32:735-45.

5. Sampayo EM, Ridgway T, Bongaerts P, Hoegh-Guldberg O. Bleaching susceptibility and mortality of corals are determined by fine-scale differences in symbiont type. Proc Natl Acad Sci USA. 2008;105:10444-9.

6. Matthews JL, Crowder CM, Oakley CA, Lutz A, Roessner U, Meyer E, et al. Optimal nutrient exchange and immune responses operate in partner specificity in the cnidarian-dinoflagellate symbiosis. Proc Natl Acad Sci USA. 2017;114:13194-9.

7. Baird AH, Guest JR, Willis BL. Systematic and biogeographical patterns in the reproductive biology of scleractinian corals. Annu Rev Ecol Evol Syst. 2009:40:551-71.

8. Kirk NL, Weis VM. Animal-Symbiodinium symbioses: foundations of coral reef ecosystems. In: Hurst C (Ed). The mechanistic benefits of microbial symbionts. Cham: Springer, Switzerland, 2016;2:269-94.

9. Fitt WK. The role of chemosensory behavior of Symbiodinium microadriaticum, intermediate hosts, and host behavior in the infection of coelenterates and molluscs with zooxanthellae. Mar Biol. 1984;81:9-17.

10. Pasternak Z, Bachr A, Abelson A, Achituv Y. Initiation of symbiosis between the soft coral Heteroxenia fuscescens and its zooxanthellae. Mar Ecol Prog Ser. 2004;279:113-6.

11. Fitt WK, Trench RK. Endocytosis of the symbiotic dinoflagellate Symbiodinium microadriaticum Freudenthal by endodermal cells of the scyphistomae of Cassiopeia xamachana and resistance of the algae to host digestion. J Cell Sci. 1983;64:195-212.

12. Wakefield TS, Farmer MA, Kempf SC. Revised description of the fine structure of in situ zooxanthellae genus Symbiodinium. Biol Bull. 2000;199:76-84.

13. Wakefield TS, Kempf SC. Development of host- and symbiont-specific monoclonal antibodies and confirmation of the origin of the symbiosome membrane in a cnidarian-dinoflagellate symbiosis. Biol Bull. 2001;200:127-43. 
14. Rosset SL, Oakley CA, Ferrier-Pagès C, Suggett DJ, Weis VM, Davy SK. The molecular language of the cnidarian-dinoflagellate symbiosis. Trends Microbiol. 2021;29:320-33.

15. Weis VM, Davy SK, Hoegh-Guldberg O, Rodriguez-Lanetty M, Pringle JR. Cell biology in model systems as the key to understanding corals. Trends Ecol Evol. 2008;23:369-76.

16. Janeway CA, Medzhitov R. Innate immune recognition. Annu Rev Immunol. 2002;20:197-216.

17. Markell DA, Trench RK, Iglesias-Prieto R. Macromolecules associated with the cell walls of symbiotic dinoflagellates. Symbiosis 1992;12:19-31.

18. Lin KL, Wang JT, Fang LS. Participation of glycoproteins on zooxanthellal cell walls in the establishment of a symbiotic relationship with the sea anemone, Aiptasia pulchella. Zool Stud. 2000;39:172-8.

19. Kvennefors EC, Leggat W, Hoegh-Guldberg O, Degnan BM, Barnes AC. An ancient and variable mannose-binding lectin from the coral Acropora millepora binds both pathogens and symbionts. Dev Comp Immunol. 2008;32:1582-92.

20. Kuniya $N$, Jimbo $M$, Tanimoto $F$, Yamashita $H$, Koike $K$, Harii $S$, et al. Possible involvement of Tachylectin-2-like lectin from Acropora tenuis in the process of Symbiodinium acquisition. Fish Sci. 2015;81:473-83.

21. Jimbo M, Yanohara $T$, Koike $K$, Koike $K$, Sakai $R$, Muramoto $K$, et al. The Dgalactose-binding lectin of the octocoral Sinularia lochmodes: characterization and possible relationship to the symbiotic dinoflagellates. Comp Biochem Physiol B Biochem Mol Biol. 2000;125:227-36.

22. Jimbo M, Yamashita H, Koike K, Sakai R, Kamiya H. Effects of lectin in the scleractinian coral Ctenactis echinata on symbiotic zooxanthellae. Fish Sci. 2010;76:355-63.

23. Helenius A, Aebi M. Intracellular functions of N-linked glycans. Science. 2001;291:2364-9.

24. Tivey TR, Parkinson JE, Mandelare PE, Adpressa DA, Peng W, Dong X, et al. Nlinked surface glycan biosynthesis, composition, inhibition, and function in cnidarian-dinoflagellate symbiosis. Micro Ecol. 2020;80:223-36.

25. Logan DD, LaFlamme AC, Weis VM, Davy SK. Flow-cytometric characterization of the cell-surface glycans of symbiotic dinoflagellates (Symbiodinium spp.). J Phycol. 2010;46:525-33.

26. Wood-Charlson EM, Hollingsworth LL, Krupp DA, Weis VM. Lectin/glycan interactions play a role in recognition in a coral/dinoflagellate symbiosis. Cell Microbiol. 2006;8:1985-93.

27. Tortorelli G, Belderok R, Davy SK, McFadden Gl, van Oppen MJH. Host genotypic effect on algal symbiosis establishment in the coral model, the anemone Exaiptasia diaphana, from the Great Barrier Reef. Front Mar Sci. 2020;6:833.

28. Dungan AM, Hartman LM, Tortorelli G, Belderok R, Lamb AM, Pisan L, et al. Exaiptasia diaphana from the Great Barrier Reef: a valuable resource for coral symbiosis research. Symbiosis. 2020;6:1-2.

29. Bay LK, Cumbo VR, Abrego D, Kool JT, Ainsworth TD, Willis BL. Infection dynamics vary between Symbiodinium types and cell surface treatments during establishment of endosymbiosis with coral larvae. Diversity. 2011;3:356-74.

30. Parkinson JE, Tivey TR, Mandelare PE, Adpressa DA, Loesgen S, Weis VM. Subtle differences in symbiont cell surface glycan profiles do not explain species-specific colonization rates in a model cnidarian-algal symbiosis. Front Microbiol. 2018;9:842.

31. Rautengarten C, Heazlewood JL, Ebert B. Profiling cell wall monosaccharides and nucleotide-sugars from plants. Curr Protoc Plant Biol. 2019;4:e20092.

32. Rautengarten $C$, Ebert $B$, Moreno I, Temple $H$, Herter $T$, Link $B$, et al. The Golg localized bifunctional UDP-rhamnose/UDP-galactose transporter family of Arabidopsis. Proc Natl Acad Sci USA. 2014;111:11563-8.

33. Rautengarten C, Ebert B, Liu L, Stonebloom S, Smith-Moritz AM, Pauly M, et al. The Arabidopsis Golgi-localized GDP-L-fucose transporter is required for plant development. Nat Commun. 2016;7:1-10.

34. Albano RM, Mourão PA. Isolation, fractionation, and preliminary characterization of a novel class of sulfated glycans from the tunic of Styela plicata (Chordata tunicata). J Biol Chem. 1986;261:758-65.

35. Levin RA, Suggett DJ, Nitschke MR, van Oppen MJH, Steinberg PD. Expanding the Symbiodinium (Dinophyceae, Suessiales) toolkit through protoplast technology. Eukaryot Microbiol. 2017;64:588-97.

36. Matthews JL, Sproles AE, Oakley CA, Grossman AR, Weis VM, Davy SK. Mentholinduced bleaching rapidly and effectively provides experimental aposymbiotic sea anemones (Aiptasia sp.) for symbiosis investigations. J Exp Biol. 2016;219:306-10.

37. R Core Team. R: a language and environment for statistical computing. 2013; 201.

38. Wickham H, Averick M, Bryan J, Chang W, McGowan L, François R, et al. Welcome to the Tidyverse. J Open Source Softw. 2019:4:1686.

39. Vidal-Dupiol J, Adjeroud M, Roger E, Foure L, Duval D, Mone Y, et al. Coral bleaching under thermal stress: putative involvement of host/symbiont recognition mechanisms. BMC Physiol. 2009;9:1-6.
40. Koike K, Jimbo M, Sakai R, Kaeriyama M, Muramoto K, Ogata T, et al. Octocoral chemical signaling selects and controls. Biol Bull. 2004;207:80-6.

41. Jimbo M, Koike K, Sakai R, Muramoto K, Kamiya H. Cloning and characterization of a lectin from the octocoral Sinularia lochmodes. Biochem Biophys Res Commun. 2005;330:157-62.

42. Jimbo M, Suda Y, Koike K, Nakamura-Tsuruta S, Kominami J, Kamei M, et al. Possible involvement of glycolipids in lectin-mediated cellular transformation of symbiotic microalgae in corals. J Exp Mar Bio Ecol. 2013;439:129-35.

43. Takeuchi $R$, Jimbo $M$, Tanimoto $F$, lijima M, Yamashita $H$, Suzuki G, et al. N-acetylD-glucosamine-binding lectin in Acropora tenuis attracts specific Symbiodiniaceae cell culture strains. Mar Drugs. 2021;19:146.

\section{ACKNOWLEDGEMENTS}

This research was funded by the Australian Government through the Australian Research Council Discovery Project DP160101539 and DP210100639. GT acknowledges receipt of the University of Melbourne International Research Scholarship and Fee Remission Scholarship, and the Botany Foundation at the University of Melbourne for awarding the Ethel McLennan prize that supported Symbiodiniaceae monosaccharide and lectin array analyses. GIMcF and $\mathrm{MJHvO}$ acknowledge Australian Research Council Discovery Grant DP160101539. MJHvO acknowledges Australian Research Council Laureate Fellowship FL180100036. BE acknowledges an ARC Future Fellowship Award FT160100276, Discovery Grant DP180102630 and support from the University of Melbourne Botany Foundation. CR acknowledges the financial aid of an Albert Shimmins COVID-19 support fund. We thank Joshua Heazlewood and Alfie Hao for the sugar nucleotide and the monosaccharide component analyses. We thank Virginia Weis, John Parkinson, Paige Mandelare and Kruti Patel for the support in the preparation of lectin array samples. We thank Ellie Cho from the Biological Optical Microscopy Platform (BOMP) at the University of Melbourne for assistance with confocal microscopy. We thank Kevin Bairos-Novak, Patrick Buerger and Wing Chan for the help with bioinformatics.

\section{AUTHOR CONTRIBUTIONS}

$\mathrm{GT}, \mathrm{BE}, \mathrm{AB}, \mathrm{SKD}, \mathrm{MJHvO}$ and GIMF contributed to the conceptual development and the final edited version of the manuscript. GT, GS, BE and CR conducted the experiments. GT performed the analyses. GT, BE, MJHvO and GIMF wrote the manuscript.

\section{COMPETING INTERESTS}

The authors declare no competing interests.

\section{ADDITIONAL INFORMATION}

Supplementary information The online version contains supplementary material available at https://doi.org/10.1038/s41396-021-01059-w.

Correspondence and requests for materials should be addressed to G.T.

Reprints and permission information is available at http://www.nature.com/ reprints

Publisher's note Springer Nature remains neutral with regard to jurisdictional claims in published maps and institutional affiliations.

Open Access This article is licensed under a Creative Commons Attribution 4.0 International License, which permits use, sharing, adaptation, distribution and reproduction in any medium or format, as long as you give appropriate credit to the original author(s) and the source, provide a link to the Creative Commons license, and indicate if changes were made. The images or other third party material in this article are included in the article's Creative Commons license, unless indicated otherwise in a credit line to the material. If material is not included in the article's Creative Commons license and your intended use is not permitted by statutory regulation or exceeds the permitted use, you will need to obtain permission directly from the copyright holder. To view a copy of this license, visit http://creativecommons. org/licenses/by/4.0/.

(c) The Author(s) 2021 